MSC 35K70, 35Q80

DOI: $10.14529 / \mathrm{mmp} 170204$

\title{
PARAMETER IDENTIFICATION AND CONTROL IN HEAT TRANSFER PROCESSES
}

\section{S.G. Pyatkov, O.V. Goncharenko}

Yugra State University, Khanty-Mansyisk, Russian Federation

E-mail: S_pyatkov@ugrasu.ru,ovg@ugrasu.ru

The article is devoted to the study of some mathematical models describing heat transfer processes. We examine an inverse problem of recovering a control parameter providing a prescribed temperature distribution at a given point of the spatial domain. The parameter is a lower order coefficient depending on time in a parabolic equation. This nonlinear problem is reduced to an operator equation whose solvability is established with the help of a priori estimates and the fixed point theorem. Existence and uniqueness theorems of solutions to this problem are stated and proved. Stability estimates are exposed. The main result is the global (in time) existence of solutions under some natural conditions of the data. The proofs rely on the maximum principle. The main functional spaces used are the Sobolev spaces.

Keywords: heat transfer; distributed control; mathematical model; parabolic equation; inverse problem; boundary value problem.

\section{Introduction}

We study the problem of recovering a lower order coefficient depending on time together with a solution in heat transfer mathematical models. This control parameter allows to ensure a given temperature distribution at a given point of spatial domain. Let $G$ be a bounded domain in $\mathbb{R}^{n}$ with boundary $\Gamma$ and $Q=(0, T) \times G$. The mathematical model can be written as

$$
\begin{gathered}
u_{t}-L_{0} u+p(t) u=f(x, t), \quad(t, x) \in Q \\
L_{0} u=A_{0} u+B_{0} u, A_{0} u=\sum_{i, j=1}^{n} \partial_{x_{i}}\left(a_{i j}(x) u_{x_{j}}\right),-B_{0} u=\sum_{i=1}^{n} a_{i}(x) u_{x_{i}}+a_{0}(x) u .
\end{gathered}
$$

Equation (1) is furnished with the initial and boundary conditions

$$
\left.u\right|_{t=0}=u_{0},\left.\quad B u\right|_{S}=g(t, x), \quad S=(0, T) \times \Gamma,
$$

where

$$
B u=\sigma\left(\sum_{i, j=1}^{n} a_{i j}(x) n_{i} u_{x_{j}}+b(x) u\right)+(1-\sigma) u,
$$

$n_{i}$ is the $i$-th coordinate of the unit outward normal to $\Gamma, \sigma(x) \in C(\Gamma)$ is a continuous function taking two values 0,1 . Thus, on different connectedness components the boundary condition can be of different type (Dirichlet, Neumann, or Robin boundary condition). Let $\Gamma_{i}=\{x \in \Gamma: \sigma(x)=i\}(i=0,1), S_{i}=(0, T) \times \Gamma_{i}$. The unknowns in (1), (2) are the solution $u$ and the function $p(t)$. The overdetermination conditions are written as

$$
u\left(x_{0}, t\right)=\psi(t) .
$$

where $\psi(t)$ is some function specified below. 
Determination of a single unknown time-dependent property such as the capacity, conductivity or diffusivity from additional local or non-local measurements of the main dependent variable at the boundary or inside the space domain represents a classical example of a coefficient identification problem (see, for instance, [1,2]). Problem (1) - (3) is classical and was studied by many authors. Numerical methods of solving the problem are developed in [3-5]. For local (in time) solvability results, see, for instance, [6, 7]. The book [8, Ch. 6] contains some abstract theory of such problems and its applications. In particular, the conditions for a local (in time) solvability of (1) - (3) are presented in Corollary 9.4.2 of [8]. Moreover, similar result is also exposed in [9]. Some results on close inverse problems can be found in [10-13]. Inverse problems with integral overdetermination conditions are studied in [14-18]. The structure of the paper is as follows. In Section 1 we formulate our results. The main result is Theorem 4 which is a global (in time) existence and uniqueness theorem for solutions to the problem (1) - (3). Some stability estimates are given in Theorem 3 . The solvability conditions are stated in terms of some inequalities and the proof relies on the maximum principle. Section 2 is devoted to the proofs of the main results.

\section{Preliminaries}

Given Banach spaces $X, Y$, the symbol $L(X, Y)$ stands for the space of linear continuous operators defined on $X$ with values in $Y$. Let $E$ be a Banach space. By $L_{p}(G ; E)$ ( $G$ is a domain in $\left.\mathbb{R}^{n}\right)$ we denote the space of strongly measurable functions defined on $G$ with values in $E$ endowed with the norm \|\|$u(x)\left\|_{E}\right\|_{L_{p}(G)}$ [19]. We employ also the spaces $C^{k}(\bar{G})$ comprising functions continuous in $G$ with all their derivatives up to order $k$ admitting continuous extensions on the closure $\bar{G}$. The Sobolev space notations are conventional, i.e., $W_{p}^{s}(G ; E), W_{p}^{s}(Q ; E)$, etc. (see the definitions in $[19,20]$ ). If $E=\mathbb{C}$ $(E=\mathbb{R})$ or $E=\mathbb{C}^{n}\left(E=\mathbb{R}^{n}\right)$ then the latter space is denoted by $W_{p}^{s}(Q)$. Similarly, we use the notations $W_{p}^{s}(G)$ or $C^{k}(\bar{G})$ rather than $W_{p}^{s}(G ; E)$ or $C^{k}(\bar{G} ; E)$. Thus, the membership $u \in W_{p}^{s}(G)$ (or $u \in C^{k}(\bar{G})$ ) for a given vector-function $u=\left(u_{1}, u_{2}, \ldots, u_{k}\right)$ means that every of its component $u_{i}$ belongs to $W_{p}^{s}(G)$ (or $C^{k}(\bar{G})$ ). The norm of the vector is just the sum of the norms of the coordinates. Given an interval $J=(0, T)$, put $W_{p}^{s, r}(Q)=$ $W_{p}^{s}\left(J ; L_{p}(G)\right) \cap L_{p}\left(J ; W_{p}^{r}(G)\right.$. Respectively, $W_{p}^{s, r}(S)=W_{p}^{s}\left(J ; L_{p}(\Gamma)\right) \cap L_{p}\left(J ; W_{p}^{r}(\Gamma)\right)$.

Consider the direct problem (1), (2). In what follows we assume that $G$ is a bounded domain in $\mathbb{R}^{n}$ with boundary $\Gamma \in C^{2}$ (see the definition in [21, p. 17]). Expose the conditions on the data of the problem. All spaces below and the coefficients of equation (1) are assumed to be real. Fix $p>n+2$ (this condition simplifies the arguments and it can be weakened). Let $q=p /(p-1)$. Denote $B_{\delta}\left(x_{0}\right)=\left\{y \in \mathbb{R}^{n}:\left|y-x_{0}\right|<\delta\right\}$.

The conditions on the coefficients of the operators $L_{0}, B$ are as follows:

$$
a_{i j} \in C^{1}(\bar{G}), a_{i}, a_{0} \in L_{p}(G), b \in C^{1}(\Gamma) .
$$

The matrix $\left\{a_{i j}\right\}$ is symmetric and the ellipticity condition

$$
\exists \delta_{1}>0: \sum_{i, j=1}^{n} a_{i j} \xi_{i} \xi_{j} \geq \delta_{1}|\xi|^{2} \forall \xi \in \mathbb{R}^{n}, x \in G,
$$

holds. The conditions on the data are of the form

$$
u_{0}(x) \in W_{p}^{2-1 / p}(G), g(t, x) \in W_{p}^{1-1 / 2 p, 2-1 / p}\left(S_{0}\right) \cap W_{p}^{1 / 2-1 / 2 p, 1-1 / p}\left(S_{1}\right),
$$




$$
\begin{gathered}
f \in L_{p}(Q),\left.B(0, x) u_{0}\right|_{\Gamma}=g(0, x) \forall x \in \Gamma, \\
u_{0}(x) \geq 0(x \in G), g(t, x) \geq 0, f \geq 0((t, x) \in Q) .
\end{gathered}
$$

We also use some additional conditions

$$
\begin{gathered}
\psi \in W_{p}^{1}(0, T), \quad \exists \delta_{2}>0:|\psi(t)| \geq \delta_{2} \forall t \in[0, T], \psi(0)=u_{0}\left(x_{0}\right), \\
\exists \delta_{0}>0: \overline{B_{\delta_{0}}\left(x_{0}\right)} \subset G, a_{i j} \in W_{p}^{2}\left(B_{\delta_{0}}\left(x_{0}\right)\right), a_{i}, a_{0} \in W_{p}^{1}\left(B_{\delta_{0}}\left(x_{0}\right)\right), \\
\nabla u_{0}(x) \in W_{p}^{2-1 / p}\left(B_{\delta_{0}}\left(x_{0}\right)\right) .
\end{gathered}
$$

Assume that $Q_{\delta}=(0, T) \times B_{\delta}\left(x_{0}\right), Q^{\gamma}=(0, \gamma) \times G$. Present some auxiliary statements. Replace the equation (1) with the equation

$$
L u=u_{t}-L_{0} u=f(x, t), \quad(t, x) \in Q .
$$

Theorem 1. Assume that conditions (4) - (7) hold. Then there exists a unique solution to (2), (12) such that $u \in W_{p}^{1,2}(Q)$. Under the additional conditions (10), (11), a solution $u$ possesses the property $\nabla u \in W_{p}^{1,2}\left(Q_{\delta}\right)$ for all $\delta<\delta_{0}$. If condition (8) is valid then the function $u$ is nonnegative in $Q$.

Proof. If $S_{0}=S$ or $S_{1}=S$ then we can refer to the standard theorems on solvability (see, for instance, [21, Theorem 9.1, Ch. 4] in the case of the Dirichlet conditions or [21, Theorem 10.4, Ch. 8] in the case of more general boundary conditions). Examine our case. First we take homogeneous initial and boundary conditions in (2). Let $D(A)=\{u \in$ $\left.W_{p}^{1,2}(Q):\left.B u\right|_{S}=0\right\}$. The claim in the case of $L_{0}=A_{0}$ results from Theorem 12.2 in [22]. In the general case the result is a consequence of Theorems 12.2 and 3.2 in [22]. To refer to Theorem 3.2 we need an additional estimate for the norm $\left\|B_{0}\right\|_{L_{\rho}\left(0, T ; L\left(E_{\theta, \infty}, E_{0}\right)\right)}$ for some $\theta \in(0,1)$ and $\rho>\max (1 /(1-\theta), p)$, where $E_{0}=L_{p}(G)$ and $E_{\theta, \infty}=\left(E_{0}, D(A)\right)_{\theta, \infty}$ is the space obtained by the real interpolation method (see the definitions [19]). Since the operator $B_{0}$ is independent of $t$, it suffices to establish the estimate

$$
\left\|B_{0} u\right\|_{L_{p}(G)} \leq c\|u\|_{W_{p}^{s}(G)}, \quad s<2
$$

and use the embedding $E_{\theta, \infty} \subset W_{p}^{s}(G)$ for $\theta>s / 2$ (which follows from the embedding (1.1) in [22] and Theorem 5.2 in [23]). This estimate results from the embedding theorems and conditions (4). We infer

$$
\left\|B_{0} u\right\|_{L_{p}(G)} \leq c\|u\|_{W_{\infty}^{1}(G)} \leq c_{1}\|u\|_{W_{p}^{s}(G)}, s \in(1+n / p, 2) .
$$

The claim of the theorem in the case of nonhomogeneous initial and boundary conditions follows from the conventional theorems on extension of the boundary conditions inside the domain (see, for instance, Theorem 7.3 in [24]).

The proof of the fact that a solution $u$ possesses the property $\nabla u \in W_{p}^{1,2}\left(Q_{\delta}\right)$ for all $\delta<\delta_{0}$ under conditions (10), (11) is realized with the finite difference method with the use of Lemma 4.6 of Ch. 2 in [25]; it is similar to that in the proof of Theorem 1.1 in [9] or in the proof of Theorem 3.1 in [26].

Different maximum principles for parabolic equations can be found in [27]. Unfortunately, they are not applicable in our case. Under stronger conditions on coefficients 
we can refer to the generalized maximum principle in [22, Theorem 17.1]. We use conventional arguments those involved in the proof of the maximum principle for generalized solutions. Let $u^{-}=\left\{\begin{array}{cl}u(t, x), & \text { if } u(t, x) \leq 0 ; \\ 0, & \text { if } u(t, x)>0 .\end{array}\right.$ Note that $u^{-} \in W_{p}^{1}(Q)$ (see [21, Sect. 4 of Ch. 2]). Moreover, we have that $\left.u^{-}\right|_{S_{0}}=0$ and $\left.u^{-}\right|_{t=0}=0$. Multiply (11) by $u^{-}$ and integrate the result over $G$. Integrating by parts and using the boundary conditions we obtain that

$$
\begin{gathered}
\frac{1}{2} \frac{d}{d t} \int_{G}\left(u^{-}\right)^{2} d x+\delta_{1} \int_{G}\left|\nabla u^{-}\right|^{2} d x+\int_{\Gamma} b\left(u^{-}\right)^{2} d \Gamma-\int_{\Gamma} g(t, x) u^{-} d \Gamma \leq \\
\leq \int_{G} f u^{-} d x+\left.\left|\int_{G} \sum_{i=1}^{n} a_{i} u_{x_{i}} u^{-}+a_{0}\right| u^{-}\right|^{2} d x \mid .
\end{gathered}
$$

Since the data are of constant sign, we derive that

$$
\frac{1}{2} \frac{d}{d t} \int_{G}\left(u^{-}\right)^{2} d x+\delta_{1} \int_{G}\left|\nabla u^{-}\right|^{2} d x \leq\left.\left|\int_{G} \sum_{i=1}^{n} a_{i} u_{x_{i}} u^{-}+a_{0}\right| u^{-}\right|^{2} d x|+| \int_{\Gamma} b\left(u^{-}\right)^{2} d \Gamma \mid .
$$

All summands on the right-hand side of (13) are estimated similarly. We use the conditions on the data, the Hölder inequality, embedding theorems, and interpolation inequalities. Estimate the summands on the right-hand side under the integral sign. We have

$$
\begin{gathered}
\left|\int_{G} a_{i} u_{x_{i}} u^{-} d x\right| \leq J_{i}(t)\left\|\nabla u^{-}\right\|_{L_{2}(G)}\left\|u^{-}\right\|_{L_{2 p /(p-2)}(G)} \leq \\
\leq c_{1} J_{i}(t)\left\|\nabla u^{-}\right\|_{L_{2}(G)}\left\|u^{-}\right\|_{W_{2}^{s}(G)}, \quad J_{i}(t)=\left\|a_{i}\right\|_{L_{p}(G)}, s=n / p .
\end{gathered}
$$

Next, the inequalities (see [19])

$$
\|u\|_{W_{2}^{s}(G)} \leq c_{2}\|u\|_{W_{2}^{1}(G)}^{s}\|u\|_{L_{2}(G)}^{1-s}, \quad|a b| \leq \varepsilon \frac{|a|^{r}}{r}+\frac{|b|^{r^{\prime}}}{r^{\prime} \varepsilon^{r^{\prime} / r}}(\varepsilon>0), \frac{1}{r}+\frac{1}{r^{\prime}}=1,
$$

imply that the right-hand side of (14) is estimated as

$$
\varepsilon\left\|\nabla u^{-}\right\|_{L_{2}(G)}^{2}+c(\varepsilon) J_{i}(t)^{2 p /(p-n)}\left\|u^{-}\right\|_{L_{2}(G)}^{2},
$$

where $2 p /(p-n) \leq p$ (since $p>n+2)$. All summands on the right-hand side of (13) except for the last of them are estimated similarly. The estimate for the last summand is simpler. We have

$$
\left|\int_{\Gamma} b\left(u^{-}\right)^{2} d \Gamma\right| \leq c\|u\|_{L_{2}(\Gamma)}^{2} \leq c_{1}\left\|u^{-}\right\|_{W_{2}^{s_{1}}(G)}^{2} \leq \varepsilon\left\|\nabla u^{-}\right\|_{L_{2}(G)}^{2}+c(\varepsilon)\left\|u^{-}\right\|_{L_{2}(G)}^{2}\left(s_{1} \in(1 / p, 1)\right) .
$$

In this case inequality (13) can be rewritten as

$$
\frac{1}{2} \frac{d}{d t} \int_{G}\left(u^{-}\right)^{2} d x+\delta_{1} \int_{G}\left|\nabla u^{-}\right|^{2} d x \leq c_{2} \varepsilon\left\|\nabla u^{-}\right\|_{L_{2}(G)}^{2}+c(\varepsilon) J_{0}(t)\left\|u^{-}\right\|_{L_{2}(G)}^{2},
$$

where $c_{2}, c(\varepsilon)$ are some positive constants and $J_{0} \in L_{1}(0, T)$. Choosing $c_{2} \varepsilon=\delta_{1}$, we arrive at the inequality

$$
y^{\prime}(t) \leq c J_{0}(t) y(t), \quad y(0)=\left.\left\|u^{-}\right\|_{L_{2}(G)}^{2}\right|_{t=0}=0 .
$$

Hence, we can conclude that $u^{-}=0$ almost everywhere in $Q$, i.e., $u \geq 0$ a.e. in $Q$. 
Corollary 1. There exists $\lambda_{0}>0$ such that $-L_{0}+\lambda$ for all $\lambda \geq \lambda_{0}$ is an isomorphism of the space $\left\{u \in W_{p}^{2}(G):\left.B u\right|_{\Gamma}=0\right\}$ onto $L_{p}(G)$ (see [22, Remark $\left.3.1(b)\right]$ ).

In view of Corollary 1 we assume below that the problem $L_{0} u=f \in L_{p}(G),\left.B u\right|_{\Gamma}=0$ has a unique solution $u \in W_{p}^{2}(G)$, otherwise we make the change of variables $u=v e^{\lambda t}$ in (1) and reduce the arguments to this case.

Theorem 2. Let conditions (4) - (7) hold. Then, for $\gamma \geq 0$, a solution $u \in W_{p}^{1,2}(Q)$ to (2), (12) with homogeneous initial and boundary conditions (i.e., $u_{0} \equiv 0, g(t, x) \equiv 0$ ) satisfies the estimate

$$
\left\|u e^{-\gamma t}\right\|_{W_{p}^{1,2}(Q)}+|\gamma|\left\|u e^{-\gamma t}\right\|_{L_{p}(Q)} \leq c\left\|f e^{-\gamma t}\right\|_{L_{p}(Q)} .
$$

Fix an arbitrary $\delta_{3}<\delta_{0}$. If conditions (10), (11) are fulfilled then the following estimate holds:

$$
\begin{aligned}
& \left\|\nabla_{x} u e^{-\gamma t}\right\|_{W_{p}^{1,2}\left(Q_{\delta_{3}}\right)}+|\gamma|\left\|\nabla_{x} u e^{-\gamma t}\right\|_{L_{p}\left(Q_{\delta_{3}}\right)} \leq \\
& c\left(\left\|\nabla_{x} f e^{-\gamma t}\right\|_{L_{p}\left(Q_{\delta_{0}}\right)}+\left\|f e^{-\gamma t}\right\|_{L_{p}(Q)}\right), \quad \gamma \geq 0 .
\end{aligned}
$$

The constant $c$ in this estimates is independent of the parameter $\gamma \geq 0$.

Proof. Let $u=v e^{\gamma t}$. Equation (12) is transformed to the equation

$$
v_{t}-L_{0} v+\gamma v=e^{-\gamma t} f(x, t) \text { with }\left.v\right|_{t=0}=0,\left.B v\right|_{S}=0 .
$$

Next, we refer to the estimate in Theorem 3.1 of [26] and make the inverse change of variables.

Remark 1. Generally speaking our reference to Theorem 3.1 in [26] is not exact, since the case of different boundary conditions on different connectedness components of the boundary is not treated there. However the proof of Theorem 3.1 remains valid in this case as well, since it is based on a partition of unity and local considerations.

Lemma 1. Let $u(t) \in W_{p}^{1}(0, T)$ and $u(0)=0$. Then there exists a constant $c>0$ independent of $\gamma>0$ such that

$$
\left\|e^{-\gamma t} u\right\|_{L_{p}(0, T)} \leq \frac{c}{\gamma}\left\|e^{-\gamma t} u_{t}\right\|_{L_{p}(0, T)} .
$$

The proof is elementary and we omit it.

Denote by $\Phi$ a solution to (12), (2) assuming that conditions (4) - (7) are fulfilled. We impose the following additional constraints on the data:

$$
\psi_{t} \leq \Phi_{t}\left(t, x_{0}\right) \text { a.e. on }(0, T), L_{0} \Phi \leq 0 \text { a.e. on } Q, \exists \delta_{4}>0: \psi(t) \geq \delta_{4} \forall t \in(0, T) \text {. }
$$

Assume that $R(\Phi)=\left\|\Phi_{i}\right\|_{L_{\infty}(Q)}+\left\|\nabla \Phi_{i}\right\|_{L_{\infty}\left(Q_{\delta_{0}}\right)}, \beta(t)=\tilde{\psi}^{\prime}(t) / \psi(t), \tilde{\psi}^{\prime}(\tau)=\psi(\tau)-$ $\Phi\left(\tau, x_{0}\right), r(t)=-\beta(t) e^{-\int_{0}^{t} \beta(\tau) d \tau}, R_{0}(\Phi)=\|r(t)\|_{L_{p}(0, T)}$.

Now we can state our main results. The former half of Theorem 3 below (the existence theorem) is known (see, for instance, [9]). However, we present here this formulation for completeness of the exposition. 
Theorem 3. Let conditions (4) - (7) and (9) - (11) hold. Then there exists a constant $\gamma_{0} \leq T$ such that on the segment $\left[0, \gamma_{0}\right]$ there exists a unique solution $(u, p)$ to $(1)-$ (3) with the property $\left.u \in W_{p}^{1,2}\left(Q^{\gamma_{0}}\right), \nabla u \in W_{p}^{1,2}\left(\left(0, \gamma_{0}\right) \times B_{\delta}\left(x_{0}\right)\right)\right)$ for all $\delta<\delta_{0}$, and $p(t) \in L_{p}\left(0, \gamma_{0}\right)$. Assume that $u_{i}(x), g_{i}(t, x), f_{i}(t, x), \psi_{i}(t)(i=1,2)$ are two different collections of the data satisfying the conditions of the theorem and the functions $\Phi_{i}(i=$ $1,2)$ are solutions to $(1),(2)$, where $p(t) \equiv 0$. Denote $r_{i}(t)=-\beta_{i}(t) e^{-\int_{0}^{t} \beta_{i}(\tau) d \tau}\left(\beta_{i}(t)=\right.$ $\left.\frac{\left(\psi_{i}^{\prime}(t)-\Phi_{i}^{\prime}\left(t, x_{0}\right)\right)}{\psi_{i}(t)}\right)$, fix a number $R>0$, and assume that $R\left(\Phi_{i}\right)+R_{0}\left(\Phi_{i}\right) \leq R(i=1,2)$. Then there exist numbers $\gamma_{0}$ and $c_{0}(R)>0$ such that there exist unique solutions $\left(u_{i}, p_{i}\right)$ $(i=1,2)$ to $(1)-(3)$ on the time segment $\left[0, \gamma_{0}\right]$ satisfying the inequalities

$$
\begin{aligned}
\| p_{1}- & p_{2} \|_{L_{p}\left(0, \gamma_{0}\right)} \leq c_{0}(R)\left(\left\|\beta_{1}-\beta_{2}\right\|_{L_{p}\left(0, \gamma_{0}\right)}+\left\|\psi_{1}-\psi_{2}\right\|_{W_{p}^{1}\left(0, \gamma_{0}\right)}+\right. \\
& +\left\|\Phi_{1}-\Phi_{2}\right\|_{L_{\infty}\left(Q^{\gamma_{0}}\right)}+\left\|\nabla\left(\Phi_{1}-\Phi_{2}\right)\right\|_{L_{\infty}\left(\left(0, \gamma_{0}\right) \times B_{\delta_{0}}\left(x_{0}\right)\right)} .
\end{aligned}
$$

Theorem 4. Let conditions (4) - (11), (15) hold. Then there exists a unique solution to (1) - (3) such that $u \in W_{p}^{1,2}(Q), p(t) \in L_{p}(0, T)$ and $\nabla u \in W_{p}^{1,2}\left(Q_{\delta}\right)$ for all $\delta<\delta_{0}$. The function $p(t)$ is nonnegative and

$$
p(t) \geq \frac{c_{0}\left(\Phi_{t}\left(t, x_{0}\right)-\psi_{t}(t)\right)}{\psi(t)}, \forall t \in[0, T]
$$

where $c_{0}$ is a positive constant depending on the norms of the data.

Remark 2. As it is easily seen, the statement of Theorem 4 remains valid if we change all signs in inequalities (8), (15), i.e., the functions $-u_{0},-g(t, x),-f(t, x),-\Phi(t, x),-\psi$ meet conditions (8), (15).

\section{Proofs of the Main Results}

Proof of Theorem 3. Let $\Phi$ be a solution to (2), (12). Make the change of variables $u=v+\Phi$. We obtain that

$$
L v+p(t)(v+\Phi)=0,\left.v\right|_{t=0}=0,\left.v\right|_{S}=0, v\left(t, x_{0}\right)=\psi(t)-\Phi\left(t, x_{0}\right)=\tilde{\psi}(t) .
$$

Next, we make the following change of variables: $v=\omega e^{-\int_{0}^{t} p(\tau) d \tau}$. We infer

$$
L \omega+p(t) e^{\int_{0}^{t} p(\tau) d \tau} \Phi=0,\left.\omega\right|_{t=0}=0,\left.\omega\right|_{S}=0, \omega\left(t, x_{0}\right)=\tilde{\psi}(t) e^{\int_{0}^{t} p(\tau) d \tau} .
$$

Put $x=x_{0}$ в (16). We arrive at the equation

$$
\tilde{\psi}^{\prime} e^{\int_{0}^{t} p(\tau) d \tau}+\psi(t) p(t) e^{\int_{0}^{t} p(\tau) d \tau}=L_{0} \omega\left(t, x_{0}\right) .
$$

Denote $\alpha(t)=e^{\int_{0}^{t} p(\tau) d \tau}$. The equations can be rewritten as

$$
\tilde{\psi}^{\prime} \alpha+\psi(t) \alpha^{\prime}=L_{0} \omega\left(t, x_{0}\right) .
$$

Expressing the function $\alpha$, we arrive at the equality

$$
\alpha(t)=e^{-\int_{0}^{t} \beta(\tau) d \tau}+\int_{0}^{t} \frac{L_{0} \omega\left(\tau, x_{0}\right)}{\psi(\tau)} e^{-\int_{\tau}^{t} \beta(\xi) d \xi} d \tau
$$


Thus, we have

$$
\alpha^{\prime}(t)=-\beta(t) e^{-\int_{0}^{t} \beta(\tau) d \tau}+\frac{L_{0} \omega\left(t, x_{0}\right)}{\psi(t)}-\beta(t) \int_{0}^{t} \frac{L_{0} \omega\left(\tau, x_{0}\right)}{\psi(\tau)} e^{-\int_{\tau}^{t} \beta(\xi) d \xi} d \tau,
$$

where the function $\omega$ is a solution to the problem

$$
L \omega=-\alpha^{\prime}(t) \Phi,\left.\omega\right|_{t=0}=0,\left.B \omega\right|_{S}=0
$$

Hence, we infer $\omega=L^{-1}\left(-\alpha^{\prime} \Phi\right)$. Denote $\alpha_{0}(t)=\alpha^{\prime}(t)$. In view of (19), (18), we derive that

$$
\begin{gathered}
\alpha_{0}(t)=r(t)+S\left(\alpha_{0}(t)\right), \quad \alpha(t)=\int_{0}^{t} \alpha_{0}(\tau) d \tau+1, \\
S\left(\alpha_{0}\right)=\frac{L_{0} \omega\left(t, x_{0}\right)}{\psi(t)}-\beta(t) \int_{0}^{t} \frac{L_{0} \omega\left(\tau, x_{0}\right)}{\psi(\tau)} e^{-\int_{\tau}^{t} \beta(\xi) d \xi} d \tau, \omega=L^{-1}\left(-\alpha_{0} \Phi\right) .
\end{gathered}
$$

Demonstrate that equation (21) is uniquely solvable in the class $\alpha_{0}(t) \in L_{p}(0, T)$. Estimate the norm of $S$. The embedding theorems (see [19]) ensure that

$$
\left|L_{0} \omega\left(t, x_{0}\right)\right| \leq c\left\|L_{0} \omega\right\|_{W_{p}^{s}\left(B_{\delta_{3}}\left(x_{0}\right)\right)}, \quad s \in(n / p, 1), \delta_{3}<\delta_{0} .
$$

Next, we consider the summands in the main part of the operator $L_{0}$. Since a function of the class $C^{\rho}(\bar{G})$ is a pointwise multiplier in $W_{q}^{s}(G)$ with $s<\rho$ (see, for instance, the item 3.3.2 in [28]), we have

$$
\left\|a_{i j} \omega_{x_{i} x_{j}}\right\|_{W_{p}^{s}\left(B_{\delta_{3}}\left(x_{0}\right)\right)} \leq c\|\omega\|_{W_{p}^{2+s}\left(B_{\delta_{3}}\left(x_{0}\right)\right)} \leq c_{1}\|\omega\|_{W_{p}^{3}\left(B_{\delta_{3}}\left(x_{0}\right)\right)}^{(2+s) / 3}\|\omega\|_{L_{p}\left(B_{\delta_{3}}\left(x_{0}\right)\right)}^{(1-s) / 3} .
$$

Lower order summands are estimated similarly. Note that the embedding theorems ensure that $a_{i}, a_{0} \in C^{1-n / p}\left(\overline{B_{\delta_{0}}\left(x_{0}\right)}\right)$. The definition of the norm in $W_{p}^{s}$ yields

$$
\begin{aligned}
& \left\|a_{i} \omega_{x_{i}}\right\|_{W_{p}^{s}\left(B_{\delta_{3}}\left(x_{0}\right)\right)} \leq c_{1}\left\|a_{i}\right\|_{W_{p}^{s}\left(B_{\delta_{3}}\left(x_{0}\right)\right)}\left\|\omega_{x_{i}}\right\|_{C\left(\overline{B_{\delta_{3}}\left(x_{0}\right)}\right)}+ \\
& +c_{1}\left\|a_{i}\right\|_{C\left(\overline{B_{\delta_{3}}\left(x_{0}\right)}\right)}\left\|\omega_{x_{i}}\right\|_{W_{p}^{s}\left(B_{\delta_{3}}\left(x_{0}\right)\right)} \leq c_{2}\|\omega\|_{W_{p}^{1+s}\left(B_{\delta_{3}}\left(x_{0}\right)\right)} \leq \\
& \leq c_{3}\|\omega\|_{W_{p}^{3}\left(B_{\delta_{3}}\left(x_{0}\right)\right)}^{(1+s) / 3}\|\omega\|_{L_{p}\left(B_{\delta_{3}}\left(x_{0}\right)\right)}^{(3-s) / 3} .
\end{aligned}
$$

Estimates $(23)-(25)$ imply that there exist constants $\theta \in(0,1)$ and $c_{4}>0$ such that

$$
\left|L_{0} \omega\left(t, x_{0}\right)\right| \leq c_{4}\|\omega\|_{W_{p}^{3}\left(B_{\delta_{3}}\left(x_{0}\right)\right)}^{\theta}\|\omega\|_{L_{p}\left(B_{\delta_{3}}\left(x_{0}\right)\right)}^{1-\theta} .
$$

Now we estimate the norm $\left\|e^{-\gamma t} S\left(\alpha_{0}\right)\right\|_{L_{p}(0, T)}$. The above definition of the operator $S$ yields

$$
\begin{gathered}
\left\|e^{-\gamma t} S\left(\alpha_{0}\right)\right\|_{L_{p}(0, T)} \leq c_{5}\left\|e^{-\gamma t} L_{0} \omega\left(t, x_{0}\right)\right\|_{L_{p}(0, T)}+c_{6}\|\beta\|_{L_{p}(0, T)} \int_{0}^{T} e^{-\gamma \tau}\left|L_{0} \omega(\tau)\right| d \tau \leq \\
\leq c_{7}\left\|e^{-\gamma t} L_{0} \omega\left(t, x_{0}\right)\right\|_{L_{p}(0, T)} .
\end{gathered}
$$

Inequalities (26), (27) and Lemma 1 imply the estimate

$$
\begin{gathered}
\left\|e^{-\gamma t} S\left(\alpha_{0}\right)\right\|_{L_{p}(0, T)} \leq c_{8}\left\|e^{-\gamma t} \omega\right\|_{L_{p}\left(0, T ; W_{p}^{3}\left(B_{\delta_{3}}\left(x_{0}\right)\right)\right.}^{\theta}\left\|e^{-\gamma t} \omega\right\|_{L_{p}\left(Q_{\delta_{3}}\right)}^{1-\theta} \leq \\
\leq \frac{c_{9}}{\gamma^{1-\theta}}\left\|e^{-\gamma t} \omega\right\|_{L_{p}\left(0, T ; W_{p}^{3}\left(B_{\delta_{3}}\left(x_{0}\right)\right)\right.}^{\theta}\left\|e^{-\gamma t} \omega_{t}\right\|_{L_{p}\left(Q_{\delta_{3}}\right)}^{1-\theta} .
\end{gathered}
$$


Next, applying Theorem 2 and the definition of $\omega$, we obtain the estimate

$$
\begin{aligned}
\left\|e^{-\gamma t} S\left(\alpha_{0}\right)\right\|_{L_{p}(0, T)} & \leq \frac{c_{10}}{\gamma^{1-\theta}}\left(\left\|e^{-\gamma t} \alpha_{0} \Phi\right\|_{L_{p}(Q)}+\left\|e^{-\gamma t} \alpha_{0} \nabla \Phi\right\|_{L_{p}\left(Q_{\delta_{0}}\right)}\right) \leq \\
& \leq \frac{c_{10}}{\gamma^{1-\theta}}\left\|e^{-\gamma t} \alpha_{0}\right\|_{L_{p}(0, T)} R(\Phi),
\end{aligned}
$$

where the constant $c_{10}$ is independent of $\gamma$ and the norms of the data, and it depends on the norms of the coefficients of the equation, the constants in embedding theorems, interpolation inequalities and $T$. Choose a constant $\gamma_{0}$ such that

$$
\frac{c_{10}}{\gamma_{0}^{1-\theta}} R(\Phi)=1 / 2
$$

In this case we have the estimate

$$
\left\|e^{-\gamma t} S\left(\alpha_{0}\right)\right\|_{L_{p}(0, T)} \leq \frac{1}{2}\left\|e^{-\gamma t} \alpha_{0}\right\|_{L_{p}(0, T)}
$$

for all $\gamma \geq \gamma_{0}$. Thus, the operator $S$ is contractive in some equivalent norm of the space $L_{p}(0, T)$ and, thereby, (21) is solvable with respect to the function $\alpha_{0}=\alpha^{\prime}(t)$. Obviously, this solution satisfies the estimate

$$
\left\|e^{-\gamma t} \alpha^{\prime}\right\|_{L_{p}(0, T)} \leq 2\left\|r(t) e^{-\gamma t}\right\|_{L_{p}(0, T)}, \gamma \geq \gamma_{0} .
$$

In particular, we infer

$$
\left\|\alpha^{\prime}\right\|_{L_{p}(0, T)} \leq 2 e^{\gamma_{0} T}\|r(t)\|_{L_{p}(0, T)}=c_{1}\left(R(\Phi), R_{0}(\Phi)\right) .
$$

We can restore the function $\alpha=1+\int_{0}^{t} \alpha_{0}(\tau) d \tau$. Given a function $\alpha$, find a solution $\omega$ to the problem (20). Fix $t_{0} \leq T$ and estimate

$$
\left\|\int_{0}^{t} \alpha^{\prime}(\tau) d \tau\right\|_{C\left(\left[0, t_{0}\right]\right)} \leq t_{0}^{1 / q}\left\|\alpha^{\prime}\right\|_{L_{p}\left(0, t_{0}\right)} \leq t_{0}^{1 / q} c_{1}\left(R, R_{0}\right) .
$$

Choose $t_{0}$ so that $t_{0}^{1 / q} c_{1}\left(R, R_{0}\right)=3 / 4$. In this case $\alpha \geq 1-3 / 4=1 / 4>0$ on $\left[0, t_{0}\right]$ and we can construct the function $\int_{0}^{t} p(\tau) d \tau=\ln (1+\alpha(t))$. Respectively, $p(t)=\alpha^{\prime}(t) /(1+\alpha(t))$. Obviously, $p(t) \in L_{p}\left(0, t_{0}\right)$. Verify that the functions $p(t), \omega(t)$ are a solution to the inverse problem (16). Integrating (19), we obtain (18) whose transformation validates equality (17) and, hence, $\tilde{\psi}^{\prime} \alpha+\tilde{\psi}(t) \alpha^{\prime}=L_{0} \omega\left(t, x_{0}\right)-\alpha^{\prime} \Phi\left(t, x_{0}\right)$. On the other hand, taking $x=x_{0}$ in $(20)$, we have $\omega^{\prime}\left(t, x_{0}\right)=L_{0} \omega\left(t, x_{0}\right)-\alpha^{\prime} \Phi\left(t, x_{0}\right)$. Hence, $\omega^{\prime}\left(t, x_{0}\right)=(\tilde{\psi} \alpha)^{\prime}$ and thereby $\omega\left(t, x_{0}\right)=\alpha \tilde{\psi}=e^{\int_{0}^{t} p(\tau) d \tau} \tilde{\psi}$, i.e., equalities (16) hold. Proceed with stability estimates. Assume that we have two collections of the data $u_{i}(x), g_{i}(t, x), f_{i}(t, x), \psi_{i}(t)(i=1,2)$ and functions $\Phi_{i}(i=1,2)$ are solutions to (1), (2) with these data and $p(t) \equiv 0$. The respective functions $\alpha_{i}$ meet the equations

$$
\alpha_{i}^{\prime}(t)=r_{i}(t)+S_{i}\left(\alpha_{i}^{\prime}(t)\right), i=1,2,
$$

where the operators $S_{i}$ are defined as in $(22)$, but instead of $\omega$ we take solutions to the problem

$$
L \omega_{i}=-\alpha_{i}^{\prime}(t) \Phi_{i}, \quad i=1,2,\left.\omega_{i}\right|_{t=0}=0,\left.B \omega_{i}\right|_{S}=0 .
$$


Thus, $\omega_{i}=L^{-1}\left(-\alpha_{i}^{\prime} \Phi_{i}\right)$ and

$$
S_{i}\left(\alpha_{i}^{\prime}\right)=\frac{L_{0} \omega_{i}\left(t, x_{0}\right)}{\psi_{i}(t)}-\beta_{i}(t) \int_{0}^{t} \frac{L_{0} \omega_{i}\left(\tau, x_{0}\right)}{\psi_{i}(\tau)} e^{-\int_{\tau}^{t} \beta_{i}(\xi) d \xi} d \tau .
$$

Choose the parameter $\gamma_{0}$ as in (29), where we take the quantity $R$ from the conditions of the theorem rather than $R(\Phi)$. Next we choose $t_{0}$ as before inserting the quantity $R$ rather than $R_{0}(\Phi)$ and $R(\Phi)$. In this case a solution $\left(u_{i}, p_{i}\right)$ to our problem satisfying the above initial and boundary data exists on the segment $\left[0, \gamma_{0}\right]\left(\gamma_{0}=t_{0}\right)$. The corresponding functions $\alpha_{1}, \alpha_{2}$ satisfy the estimate

$$
\alpha_{i}(t) \geq 1 / 4, \quad\left\|\alpha_{i}\right\|_{W_{p}^{1}(0, T)} \leq c(R)
$$

We have

$$
\begin{aligned}
& S_{1}\left(\alpha_{1}^{\prime}\right)- S_{1}\left(\alpha_{2}^{\prime}\right)=\frac{L_{0}\left(\omega_{1}-\omega_{2}\right)\left(t, x_{0}\right)}{\psi_{1}(t)}+L_{0} \omega_{2}\left(\frac{1}{\psi_{1}(t)}-\frac{1}{\psi_{2}(t)}\right)- \\
&- \beta_{1}(t) \int_{0}^{t} \frac{L_{0}\left(\omega_{1}-\omega_{2}\right)\left(\tau, x_{0}\right)}{\psi_{1}(\tau)} e^{-\int_{\tau}^{t} \beta_{1}(\xi) d \xi} d \tau- \\
&-\int_{0}^{t} L_{0} \omega_{2}\left(\tau, x_{0}\right)\left(\frac{\beta_{1}(t) e^{-\int_{\tau}^{t} \beta_{1}(\xi) d \xi}}{\psi_{1}(\tau)}-\frac{\beta_{2}(t) e^{-\int_{\tau}^{t} \beta_{2}(\xi) d \xi}}{\psi_{2}(\tau)}\right) d \tau .
\end{aligned}
$$

Subtracting equalities (31) for $i=1,2$, we infer

$$
L \omega_{1}-L \omega_{2}=L\left(\omega_{1}-\omega_{2}\right)=-\left(\alpha_{1}^{\prime}(t)-\alpha_{2}^{\prime}(t)\right) \Phi_{1}+\alpha_{2}^{\prime}\left(\Phi_{1}-\Phi_{2}\right) .
$$

Repeating the arguments those in the proof of (28), we obtain the inequality

$$
\begin{gathered}
\left\|e^{-\gamma t} L_{0}\left(\omega_{1}-\omega_{2}\right)\left(t, x_{0}\right)\right\|_{L_{p}\left(0, \gamma_{0}\right)} \leq \frac{c_{10}}{\gamma^{1-\theta}}\left(\left\|e^{-\gamma t}\left(\alpha_{1}^{\prime}-\alpha_{2}^{\prime}\right)\right\|_{L_{p}\left(0, \gamma_{0}\right)} c(R)+\right. \\
\left.+c_{1}(R)\left(\left\|\Phi_{1}-\Phi_{2}\right\|_{L_{\infty}\left(Q^{\gamma_{0}}\right)}+\left\|\nabla\left(\Phi_{1}-\Phi_{2}\right)\right\|_{L_{\infty}\left(\left(0, \gamma_{0}\right) \times B_{\delta_{0}}\left(x_{0}\right)\right)}\right)\right) .
\end{gathered}
$$

The claim of the theorem follows from (32) - (34), the equality $\alpha_{1}^{\prime}-\alpha_{2}^{\prime}=r_{1}-r_{2}+S_{1}\left(\alpha_{1}^{\prime}\right)-$ $S_{1}\left(\alpha_{2}^{\prime}\right)$, and some simplest estimates.

Proof of Theorem 4. Let the conditions of the theorem hold. As in Theorem 3 we reduce the problem to the study of equation (21) and justify its solvability. Since the norm of $S$ is less than 1 and the operator is linear, a solution to (21) can be found using the method of successive approximations beginning with $\alpha^{0}=0$. Successive approximations are written as

$$
\alpha^{i}(t)=r(t)+S\left(\alpha^{i-1}(t)\right) .
$$

In view of (15), $\alpha^{1}=r(t) \geq 0$. Assume that the function $\alpha^{i-1}$ is nonnegative. Demonstrate that $\alpha^{i}$ is nonnegative too. The corresponding function $\omega^{i}$ is a solution to (20), i.e., we have

$$
L \omega^{i}=-\alpha^{i-1} \Phi,\left.\omega^{i}\right|_{t=0}=0,\left.B \omega^{i}\right|_{S}=0 .
$$

Since condition (8) is fulfilled, by Theorem $1 \Phi \geq 0$ a.e. in $Q$. By condition $L_{0} \Phi \leq 0$ a.e. and $\alpha^{i-1} \geq 0$ a.e. Hence, $L_{0}\left(-\alpha^{i-1} \Phi\right) \geq 0$ a.e. Consider the problem

$$
L \omega^{*}=-\alpha^{i-1} L_{0} \Phi,\left.\omega^{*}\right|_{t=0}=0,\left.B \omega^{*}\right|_{S}=\left.\alpha^{i-1} B \Phi\right|_{S}=\alpha^{i-1} g(t, x) \geq 0 .
$$


By Theorem 1 a solution $\omega^{*}$ is nonnegative in $Q$. Define the function $\tilde{\omega}=L_{0}^{-1} \omega^{*}$. We have $\left.\tilde{\omega}\right|_{t=0}=0,\left.B \tilde{\omega}\right|_{S}=0$ (by construction), $\tilde{\omega} \in W_{p}^{1,2}(Q)$, and $L_{0} \tilde{\omega} \in W_{p}^{1,2}(Q)$. Moreover, we can conclude that

$$
\omega_{t}^{*}-L_{0}\left(\omega^{*}-\alpha^{i-1} \Phi\right)=0 .
$$

Applying $L_{0}^{-1}$ to this equality, we can claim that $\tilde{\omega}_{t}-\left(L_{0} \tilde{\omega}-\alpha^{i-1} \Phi\right)=0$. Hence, $\tilde{\omega}_{t}-L_{0} \tilde{\omega}=$ $-\alpha^{i-1} \Phi$. In view of the uniqueness theorem, we derive that $\omega^{i}=\tilde{\omega}$ and thus $L_{0} \omega^{i}=\omega^{*} \geq 0$ a.e. Consider equality (35) and recall the definition of $S$ (see (22)):

$$
S\left(\alpha^{i-1}\right)=\frac{L_{0} \omega^{i}\left(t, x_{0}\right)}{\psi(t)}-\beta(t) \int_{0}^{t} \frac{L_{0} \omega^{i}\left(\tau, x_{0}\right)}{\psi(\tau)} e^{-\int_{\tau}^{t} \beta(\xi) d \xi} d \tau .
$$

Since $\beta(t) \leq 0$, every summand here is nonnegative and in view of (35), $\alpha^{i} \geq 0$ a.e. Since the limit $\alpha_{0}$ is a strong limit of the sequence $\alpha^{i}$ in the space $L_{p}(0, T)$, we conclude that $\alpha_{0} \geq 0$ a.e. In this case the function $\int_{0}^{t} p(\tau) d \tau=\ln \left(\int_{0}^{t} \alpha_{0}(\tau) d \tau+1\right)$ is defined on the whole segment $[0, T]$, respectively the function $p(t)=\frac{\alpha^{\prime}}{1+\alpha(t)}$ is a solution to our problem. Establish the desired estimate. We have (see (30)) that

$$
\int_{0}^{t} p(\tau) d \tau=\int_{0}^{t} \frac{\alpha^{\prime}(\tau)}{1+\alpha(\tau)} d \tau \leq T^{1 / q}\left\|\alpha^{\prime}\right\|_{L_{p}(0, T)} \leq c_{1}\left(R, R_{0}\right) T^{1 / q}, q=p /(p-1) .
$$

From (21), it follows that $\alpha^{\prime}(t)=p(t) e^{\int_{0}^{t} p(\tau) d \tau} \geq r(t) \geq 0$. Thus,

$$
p(t) \geq r(t) / T^{1 / q} c_{1}\left(R, R_{0}\right)=r(t) c_{0}, t \in[0, T] .
$$

The authors were supported by the Russian Foundation for Basic Research and the Government of KhMAO-Yugra (Grant No. 15-41-00063, r_ural_a).

\section{References}

1. Alifanov O.M. Inverse Heat Transfer Problems. Berlin, Heidelberg, Springer-Verlag, 1994. DOI:10.1007/978-3-642-76436-3

2. Ozisik M.N., Orlando H.R.B. Inverse Heat Transfer. N.-Y., Taylor \& Francis, 2000.

3. Dehghan M. Numerical Computation of a Control Function in a Partial Differential Equation. Applied Mathematics and Computation, 2004, vol. 147, no. 2, pp. 397-408. DOI: 10.1016/S0096-3003(02)00733-6

4. Dehghan M., Shakeri F. Method of Lines Solutions of the Parabolic Inverse Problem with an Overspecification at a Point. Numerical Algorithms, 2009, vol. 50, no. 4, pp. 417-437. DOI: $10.1007 / \mathrm{s} 11075-008-9234-3$

5. Dehghan M. Parameter Determination in a Partial Differential Equation from the Overspecified Data. Mathematical and Computer Modelling, 2005, vol. 41, no. 2-3, pp. 196-213. DOI: $10.1016 /$ j.mcm.2004.07.010

6. Iskenderova A.D., Akhundov A.Ya. Inverse Problem for a Linear System of Parabolic Equations. Doklady Mathematics, 2009, vol. 79, no. 1, pp. 73-75. DOI:10.1134/S1064562409010219

7. Kuliev M.A. A Multidimensional Inverse Problem for a Parabolic Equation in a Bounded Domain. Nonlinear Boundary Value Problems, 2004, vol. 14, pp. 138-145. 
8. Prilepko A.I., Orlovsky D.G., Vasin I.A. Methods for Solving Inverse Problems in Mathematical Physics. N.-Y., Marcel Dekker, 1999.

9. Pyatkov S.G., Samkov M.L. On Some Classes of Coefficient Inverse Problems for Parabolic Systems of Equations. Siberian Advances in Mathematics, 2012, vol. 22, no. 4, pp. 287-302. DOI:10.3103/S1055134412040050

10. Cannon J.R., Yin H.-M. A Class of Non-Linear Non-Classical Parabolic Equations. Journal of Differential Equations, 1989, vol. 79, issue 2, pp. 266-288. DOI:10.1016/0022-0396(89)90103-4

11. Shidfar A. An Inverse Heat Conduction Problem. Southeast Asian Bulletin of Mathematics, 2003, vol. 26, no. 3, pp. 503-507. DOI:10.1007/s10012-002-0503-0

12. Ivanchov N.I., Pabyrivska N.V. On Determination of Two Time-Dependent Coefficients in a Parabolic Equation. Siberian Mathematical Journal, 2002, vol. 43, no. 2, pp. 323-329. DOI:10.1023/A:1014749222472

13. Ivanchov M. Inverse Problems for Equations of Parabolic Type. Lviv, WNTL Publishers, 2003.

14. Cannon J.R. An Inverse Problem of Finding a Parameter in a Semi-linear Heat Equation. Journal of Mathematical Analysis and Applications, 1990, vol. 145, issue 2, pp. 470-484. DOI:10.1016/0022-247X(90)90414-B

15. Kozhanov A.I. Parabolic Equations with an Unknown Time-Dependent Coefficient. Computational Mathematics and Mathematical Physics, 2005, vol. 45, no. 12, pp. 2085-2101.

16. Ismailov M.I., Kanca F. Inverse Problem of Finding the Time-Dependent Coefficient of Heat Equation from Integral Overdetermination Condition Data. Inverse Problems In Science and Engineering, 2012, vol. 20, issue 2, pp. 463-476. DOI:10.1080/17415977.2011.629093

17. Hussein M.S., Lesnic D. Simultaneous Determination of Time-Dependent Coefficients and Heat Source. International Journal for Computational Methods in Engineering Science and Mechanics, 2016, vol. 17, issue 5-6, pp. 401-411. DOI:10.1080/15502287.2016.1231241

18. Kamynin V.L. Unique Solvability of the Inverse Problem of Determination of the Leading Coefficient in a Parabolic Equation. Differential Equations, 2011, vol. 47, no. 1, pp. 91-101. DOI:10.1134/S0012266111010101

19. Triebel H. Interpolation Theory. Function Spaces. Differential Operators. Berlin, VEB Deutscher verlag der wissenschaften, 1978. DOI:10.1002/zamm.19790591227

20. Denk R., Hieber M., Prüss J. Optimal $L_{p}-L_{q}$-estimates for Parabolic Boundary Value Problems with Inhomogeneous Data. Mathematische zeitschrift, 2007, vol. 257, issue 1, pp. 193-224. DOI:10.1007/s00209-007-0120-9

21. Ladyzhenskaya O.A., Solonnikov V.A., Ural'tseva N.N. Linear and Quasilinear Equations of Parabolic Type. American Mathematical Society, 1968.

22. Amann H. Nonautonomous Parabolic Equations Involving Measures. Journal of Mathematical Sciences, 2005, vol. 130, no. 4, pp. 4780-4802. DOI:10.1007/s10958-005-0376-8

23. Amann H. Nonhomogeneous Linear and Quasilinear Elliptic and Parabolic BoundaryValue Problems. Function Spaces, Differential Operators and Nonlinear Analysis, Stuttgart, Teubner, 1993, vol. 133, pp. 9-126.

24. Grisvard P. Equations differentielles abstraites. Annales scientifiques de l'Ecole Normale Superieure, 1969, vol. 2, issue 2, pp. 311-395.

25. Ladyzhenskaya O.A., Ural'tseva N.N. Linear and Quasilinear Elliptic Equations. N.-Y., London, Academic Press, 1968. 
26. Pyatkov S.G., Tsybikov B.N. On Some Classes of Inverse Problems for Parabolic and Elliptic Equations. Journal of Evolution Equations, 2011, vol. 11, no. 1, pp. 155-186. DOI:10.1007/s00028-010-0087-6

27. Lieberman G.M. Second Order Parabolic Differential Equations. Singapore, World Scientific Publishing, 1998.

28. Triebel H. Theory of Function Spaces. Basel, Boston, Stuttgart, Birkhauser verlag, 1983. DOI: $10.1007 / 978-3-0346-0416-1$

Received March 4, 2017

УДК 517.95

DOI: $10.14529 / \mathrm{mmp} 170204$

\title{
ОПРЕДЕЛЕНИЕ ПАРАМЕТРА И УПРАВЛЕНИЕ В ПРОЦЕССАХ ТЕПЛОПЕРЕНОСА
}

\section{С.Г. Пятков, О.В. Гавриленко}

Югорский государственный университет, г. Ханты-Мансийск

\begin{abstract}
Статья посвящена изучению некоторьх математических моделей, описывающих процессы теплопереноса. Мы рассматриваем обратную задачу о восстановлении управляющего параметра, который обеспечивает заданное температурное распределение в данной точке пространственной области. Данный параметр - есть младший коэффициент в параболическом уравнении, зависящий от времени. Эта нелинейная задача сводится к операторному уравнения, разрешимость которого устанавливается при помощи априорных оценок и теоремы о неподвижной точке. Сформулированы и доказаны теоремы существования и единственности решений этой задачи. Установлены оценки устойчивости. Главный результат - глобальная по времени теорема существования решений при некоторых естественных условиях на данные задачи. Доказательство опирается на принцип максимума. Используемые функциональные пространства - пространства Соболева.

Ключевые слова: теплоперенос; распределенное управление; математическая модель; параболическое уравнение; обратная задача; краевая задача.
\end{abstract}

Сергей Григорьевич Пятков, доктор физико-математических наук, профессор, кафедра высшей математики, Югорский государственный университет (г. ХантыМансийск, Российская Федерация), S_pyatkov@ugrasu.ru.

Олег Валериевич Гончаренко, аспирант, кафедра высшей математики, Югорский государственный университет (г. Ханты-Мансийск, Российская Федерация), ovg@ugrasu.ru.

Поступила в редакиию 4 марта 2017 г. 